\title{
0.75 atoms improve the clock signal of 10,000 atoms
}

I. Kruse, K. Lange, J. Peise, B. Lücke, L. Pezzè, et al.

\section{SPIE.}




\title{
0.75 atoms improve the clock signal of 10,000 atoms
}

\author{
I. Kruse ${ }^{\mathrm{a}}$, K. Lange ${ }^{\mathrm{a}}$, J. Peise ${ }^{\mathrm{a}}$, B. Lücke ${ }^{\mathrm{a}}$, L. Pezzè̀ ${ }^{\mathrm{b}}$, J. Arlt ${ }^{\mathrm{c}}$, W. Ertmer ${ }^{\mathrm{a}}$, C. Lisdat ${ }^{\mathrm{d}}$, L. \\ Santos ${ }^{\mathrm{e}}$, A. Smerzi ${ }^{\mathrm{b}}$, and C. Klempt ${ }^{\mathrm{a}}$ \\ anstitut für Quantenoptik, Leibniz Universität Hannover, Welfengarten 1, D-30167 Hannover, \\ Germany \\ ${ }^{\mathrm{b}}$ QSTAR, INO-CNR and LENS, Largo Enrico Fermi 2, I-50125, Firenze, Italy \\ ${ }^{\mathrm{C}}$ Institut for Fysik og Astronomi, Aarhus Universitet, Ny Munkegade 120, DK-8000 Arhus C, \\ Denmark \\ dPhysikalisch-Technische Bundesanstalt, Bundesallee 100, D-38116 Braunschweig, Germany \\ eInstitut für Theoretische Physik, Leibniz Universität Hannover, Appelstraße 2, \\ D-30167 Hannover, Germany
}

\begin{abstract}
Since the pioneering work of Ramsey, atom interferometers are employed for precision metrology, in particular to measure time and to realize the second. In a classical interferometer, an ensemble of atoms is prepared in one of the two input states, whereas the second one is left empty. In this case, the vacuum noise restricts the precision of the interferometer to the standard quantum limit (SQL). Here, we propose and experimentally demonstrate a novel clock configuration that surpasses the SQL by squeezing the vacuum in the empty input state. We create a squeezed vacuum state containing an average of 0.75 atoms to improve the clock sensitivity of 10,000 atoms by $2.05_{-.37}^{+.34} \mathrm{~dB}$. The SQL poses a significant limitation for today's microwave fountain clocks, which serve as the main time reference. We evaluate the major technical limitations and challenges for devising a next generation of fountain clocks based on atomic squeezed vacuum.
\end{abstract}

Keywords: Atomic clock, squeezed vacuum, precision below the SQL

\section{INTRODUCTION}

Precision measurements allow to probe the boundaries of our understanding of physics. Prominent recent examples include the discovery of gravitational waves with optical interferometers ${ }^{1}$ and the improving bounds on the drift of fundamental constants with atomic interferometers. ${ }^{2,3}$ The current generation of atomic and optical interferometers is however fundamentally limited by vacuum noise, the so-called standard quantum limit (SQL). Squeezing the vacuum entering one port of an optical gravitational wave detector was proposed in the $80 \mathrm{~s}^{4}$ to surpass the SQL when measuring the length difference between two optical paths. Can squeezed vacuum be useful to improve the measurement of time? Up to now, the concept of vacuum squeezing has not been transferred to atomic clocks or atom interferometry in general.

In following, we design and implement an atom interferometer in clock configuration which exploits atomic squeezed vacuum. The principle of sub-shot-noise interferometry is introduced before the experimental realization of the sub-shot-noise frequency standard is discussed. The clock is operated by combining $N=10^{4}$ atoms in one input state with a quadrature-squeezed vacuum with an average of 0.75 atoms in the second input state. The squeezed vacuum is generated by spin-changing collisions in a Bose-Einstein condensate of neutral ${ }^{87} \mathrm{Rb}$ atoms - in direct analogy to optical parametric down-conversion. ${ }^{5-7}$ In contrast to existing methods ${ }^{8-17}$ to increase the sensitivity of atomic clocks beyond the SQL in large ensembles, our concept disentangles the challenge of increasing the number of atoms (in the main input state) from the creation of squeezing (in the vacuum state). In particular, the vacuum state remains weakly populated during its preparation, making it immune to losses. These central advantages are also exploited in squeezed-vacuum optical interferometers for the detection of gravitational waves, as demonstrated in GEO600 ${ }^{18}$ and LIGO, ${ }^{19}$ where coherent states of $>10 \mathrm{~W}$ are combined with a low-power squeezed vacuum state to achieve sub-SQL measurement uncertainty.

Further author information: Correspondence should be addressed to C.K. (email:klempt@iqo.uni-hannover.de) 

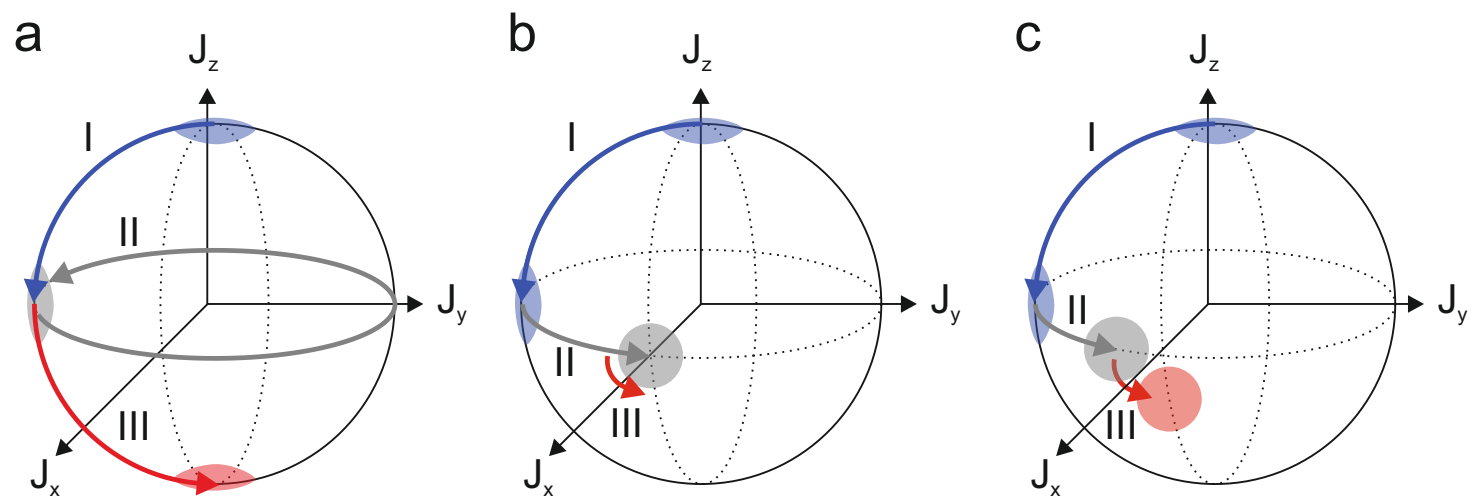

Figure 1. Ramsey measurements with different phases $\Delta \phi$. a/b/c The measurement starts with the atomic ensemble prepared in the ground state corresponding to the north pole of the Bloch sphere. I: A $\pi / 2$ pulse rotates the state around the $J_{x}$ axis to the equator. II: The phase evolution of $\Delta \phi=\Delta \omega t$ during the time $t$ depends on the detuning $\Delta \omega$ and is depicted by a rotation around the $J_{z}$ axis. III: A final $\pi / 2$ pulse, again corresponding to a rotation around $J_{x}$ transfers the phase shift to a population imbalance of the ground and exited state. a A phase evolution of $2 \pi$ leads to a complete transfer to the exited state, corresponding to the south pole of the sphere. $\mathbf{b}$ After a phase evolution of $1.5 \pi$ the state rests on the $J_{x}$ axis corresponding to a perfect $50: 50$ superposition after the rotation. c If the rotation is applied after a phase evolution of $1.25 \pi$ the resulting state shows a population imbalance of the ground and exited states. For perfect $\pi / 2$ rotations, the resulting state will always be in the $J_{y}$-plane.

\section{ATOMIC INTERFEROMETERS BELOW THE STANDARD QUANTUM LIMIT}

Since the second was redefined with respect to the microwave transition between two hyperfine levels of the ground state of ${ }^{133} \mathrm{Cs}$ in 1960, the stability and accuracy of atomic clocks has improved tremendously. Nowadays, microwave clocks operate with stabilities in the $10^{-16}$ regime. ${ }^{20}$ The stability can be improved further by employing transitions with higher frequencies such as optical transitions. By eliminating noise sources in the environment and monitoring all sources that cannot be completely suppressed, such as blackbody radiation, it was recently possible to achieve a stability of $6.4 \cdot 10^{-18}$ in an optical ${ }^{133} \mathrm{Cs}$ lattice clock. ${ }^{21}$

However, the stability of all interferometers, including atomic clocks, with classical input states is fundamentally limited due to the shot-noise limit. In 1999, the first fundamentally limited microwave clock with a stability of $4 \cdot 10^{-14}$ was realized ${ }^{22}$ and today the best microwave clocks are limited by the shot-noise limit. In the future, this limit will also be a restriction for optical clocks. However, it can be overcome with interferometers that employ squeezed states or more complicated entangled states instead of classical input states.

\subsection{Atomic clocks}

In order to operate a frequency standard different constituents need to be realized. A frequency reference is needed as well as an oscillator to probe this reference and a detector to analyze the signal. Depending on this signal a control loop gives a feed back to the probing oscillator. Such a frequency standard can be as a definition for time, if the frequency is suitably divided. In 1967 the second was redefined from the astronomical standard, referenced to the mean length of a solar day, to the atomic standard, referenced to a microwave transition between hyperfine-levels of ${ }^{133} \mathrm{Cs}$ at $0 \mathrm{~K}$.

The primary ${ }^{133} \mathrm{Cs}$ standard is considered a passive frequency standard since it acts as the reference which is probed by an external oscillator. Active frequency standards on the other hand are those where the standard itself acts as the oscillator, for example an active hydrogen maser. While cesium is defined as the primary frequency standard many other atomic species can be used to act as a secondary frequency standard, that don't necessarily have to have a larger uncertainty.

The most commonly used probing method was first introduced by Ramsey in $1949 .{ }^{23}$ An ensemble of two-level atoms with ground state $|0\rangle$ and exited state $|1\rangle$ is prepared in the ground state. A $\pi / 2$ pulse is used to create a superposition of the two states. This can be achieved by either driving a single or a two-photon transition. This is depicted on the Bloch sphere in Fig. 1 and corresponds to a rotation around the $J_{x}$ axis. An evolution time 
a

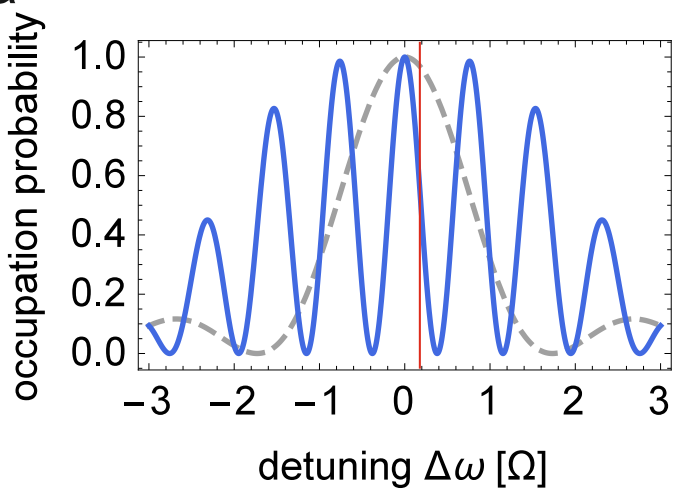

b

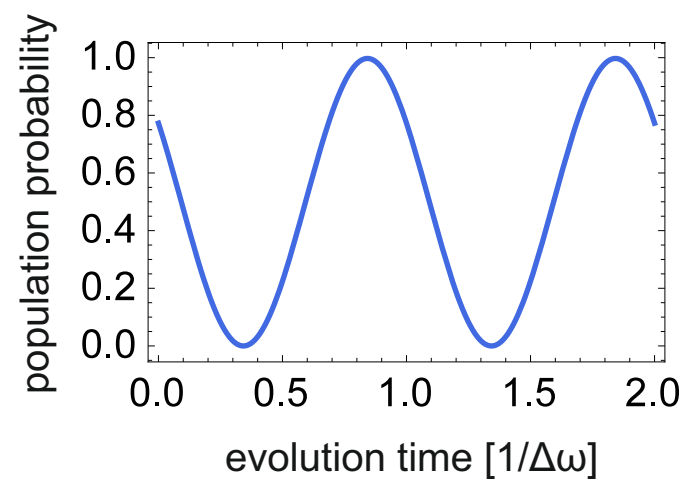

Figure 2. Ramsey measurements for different detunings $\Delta \omega$ and evolution times $t$. a Population probability of the exited state $|1\rangle$ as a function of the detuning $\Delta \omega$. The gray dashed line represents a Rabi transition, while the blue line is calculated for an evolution time $t$ of $1 / \Omega$. b Population probability of the exited state $|1\rangle$ as a function of the evolution time $t$. The detuning is fixed to $0.5 \Omega$.

$t$ follows, during which a phase shift is imprinted onto the ensemble. The phase shift depends on the detuning of the $\pi / 2$ pulse $\Delta \omega$ from the resonance of the transition and the evolution time as $\Delta \phi=\Delta \omega t$. In the Bloch sphere picture, this corresponds to a rotation around $J_{z}$ such that a phase shift $\Delta \phi>2 \pi$ is depicted by multiple rotations around $J_{z}$. A second $\pi / 2$ pulse is applied, which maps the phase shift $\Delta \phi$ onto a population imbalance between the ground and exited state. This is again depicted by a rotation around $J_{x}$ on the Bloch sphere. If the rotation is perfect i.e. it reaches the south pole of the sphere, this corresponds to a complete transfer of the population to $|1\rangle$ for $\Delta \phi=n 2 \pi$, where $n$ is an integer (Fig. 1a). If $\Delta \phi=(1+2 n) \pi$, the atoms are completely transferred back to the ground state $|0\rangle$ of the system. If $\Delta \phi=(1 / 2+n) \pi$, the state rests on the $J_{x}$ axis and is therefore in a perfect 50:50 superposition after the rotation (Fig. 1b). Any phase in between leads to an imperfect transfer resulting in a population imbalance which corresponds to a state vector pointing in between the equator and the poles (Fig. 1c). For perfect $\pi / 2$-pulses, the complete three-part sequence can be expressed as a single rotation around $J_{y}$.

This measurement technique has the advantage that the fringe spacing, and therefore the slope of the population probability as a function of the detuning $\Delta \omega$, can be adjusted by the evolution time $t$. This is shown in Fig. 2a, where a Rabi measurement, without any evolution time (dashed gray) is compared to a Ramsey measurement with an evolution time of $t=1 / \Omega$, where $\Omega$ is the undetuned Rabi frequency of the transition. Fig. $2 \mathrm{~b}$ shows the population probability of the exited state in dependence on the evolution time $t$. For small evolution times, the population probability oscillates with a sinusoidal function. For longer evolution times, the system will start to decohere leading to a damping and a loss of contrast.

\subsection{Sub-shot-noise interferometry}

The phase sensitivity of the interferometer can be defined as

$$
\Delta \phi=\frac{\Delta P}{\partial\langle P\rangle / \partial \phi}
$$

where $P=N_{0} / N$ is the transferred fraction with $N_{0}$ being the number of atoms in the ground state and $N$ the total number of atoms. $\partial\langle P\rangle / \partial \phi$ is the slope of the Ramsey measurement. For uncorrelated particles or successive measurements, $N_{0}$ follows a binomial distribution with standard deviation $\Delta N_{0}=\sqrt{N_{0}\left(1-N_{0} / N\right)}$. This fundamental minimal error for $N$ uncorrelated particles or measurements is called the shot noise limit. It can be reduced by increasing the number of particles or the number of measurements. If the central fringe can now be approximated by a sinusoidal function, the slope and standard deviation cancel, such that the phase sensitivity only depends on the number of particles $N$ and not on the phase or the detuning, with $\Delta \phi=\frac{1}{\sqrt{N}}$. 
In practice, the system will experience additional technical noise. Since the overall noise is divided by the slope of the signal in Eq. (1), technical noise sources are best suppressed at the middle position of a Ramsey fringe as indicated in red in Fig. 2a.

While the shot-noise limit restricts the measurement precision for uncorrelated states, it can be surpassed by correlated states, for example with spin squeezed states. These measurements are then restricted by a more fundamental limit, the Heisenberg limit. This limit is a direct consequence of the Heisenberg uncertainty $\Delta \phi \Delta\left(N_{1}-N_{0}\right) \geq 1$, where $\Delta \phi=\Delta\left(\phi_{1}-\phi_{0}\right)$ is the variance of the relative phase between the two outputs. If one now assumes the largest possible fluctuations in the particle number difference $N$, the Heisenberg limit

$$
\Delta \phi \geq 1 / N
$$

follows. The spin squeezing parameter $\xi$ defines the improvement, a squeezed state can have in a Ramsey measurement as $\Delta \phi=\xi / \sqrt{N}<1 / \sqrt{N}$.

The shot-noise limit has been surpassed in multiple experiments by populating both interferometer states symmetrically with an entangled many body state. ${ }^{8,14,15,17}$ Interferometric sub-shot noise measurements of physical properties include a magnetometer showing a sensitivity $2 \mathrm{~dB}$ below shot-noise is presented in Ref. ${ }^{24}$ The state preparation relies on collective spin squeezing via a QND measurement in laser cooled ${ }^{87} \mathrm{Rb}$. Another magnetometer with a suppression of fluctuations of $3.8 \mathrm{~dB}$ is realized by employing a one-axis twisting Hamiltonian. ${ }^{25}$ Other magnetometers include Ref. ${ }^{9}$ Ref. $^{26}$ demonstrates the mapping of a microwave field with a sensitivity of $4 \mathrm{~dB}$ below the standard quantum limit. This enhancement is again enabled by using spin squeezed input states that are prepared via the non-linear interactions of a one-axis twisting Hamiltonian. A measurement of a frequency standard $1.1 \mathrm{~dB}$ below the shot noise limit has been shown in Ref. ${ }^{27}$ The state was prepared with a quantum non-demolition measurement. In Ref. ${ }^{28}$ a frequency standard operating $0.57 \mathrm{~dB}$ below shot noise has been realized in a system of two entangled trapped ${ }^{9} \mathrm{Be}^{+}$ions.

Enhancing the interferometer by initializing both input ports with an equally divided entangled ensemble has the disadvantage, that the generation of the input state typically highly depends on the number of atoms and the procedure might therefore not be easily scalable. This competes with the idea of increasing the number of particles to enhance the interferometric sensitivity. The following section presents an alternative approach which circumvents the problem of the varying state preparation for varying particle numbers. The interferometer performance is enhanced by a squeezed vacuum state instead of acting on both input state. ${ }^{4}$ This technique is commonly used in optics as for example at the GEO 600 gravitational wave observatory. ${ }^{29}$

\section{RESULTS}

Our atomic clock consists of a four-mode linear Ramsey interferometer when described in terms of the Zeeman states $| \pm 1\rangle=\left|F=1, m_{F}= \pm 1\right\rangle,|0\rangle=\left|F=1, m_{F}=0\right\rangle$ and $|e\rangle=\left|F=2, m_{F}=0\right\rangle$ (see Fig. 3). It can be reduced to a standard two-level Ramsey sequence in terms of the magnetically insensitive clock states $|g\rangle=\frac{|+1\rangle+|-1\rangle}{\sqrt{2}}$ and $|e\rangle$ (see Fig. 3 and $^{30}$ ). The atoms are prepared in a balanced superposition of $|0\rangle$ and $| \pm 1\rangle$ by a radio-frequency (rf) $\pi / 2$ pulse, which couples the states $|0\rangle$ and $|g\rangle$ (pulse I in Fig. 3a). A subsequent microwave (mw) $\pi$ pulse transfers the atoms from $|0\rangle$ to $|e\rangle$ (II). In this superposition state, the atoms sense the evolution of time by acquiring a phase shift $\theta=2 \pi \tau \delta$, which depends on the detuning $\delta$ of the employed microwave oscillator and the phase evolution time $\tau=\tau_{\mathrm{R}}+\tau_{\mathrm{mw}}$, where $\tau_{\mathrm{R}}$ is the Ramsey interrogation time and $\tau_{\mathrm{mw}}$ is the microwave pulse duration. A second mw $\pi$ pulse transfers atoms back in $|0\rangle$ (III). Finally, a second rf pulse (IV) closes the interferometer. The phase is estimated from the fraction $f=\frac{N_{g}}{N} \approx \frac{N_{g}}{N_{g}+N_{0}} \approx \frac{N_{+1}+N_{-1}}{N}$ of atoms in the output state $|g\rangle$, which can be obtained directly from an absorption image of all three Zeeman components. These Zeeman components are spatially separated by a magnetic gradient during free fall. Subsequently, absorption imaging is performed on the closed transition $F=2 \rightarrow F^{\prime}=3$ on the Rb D2 line with a resonant laser beam derived from an external-cavity diode laser. Atoms in $F=1$ are pumped to the cycling transition by an independent repumping laser beam on the transition $F=1 \rightarrow F^{\prime}=2$. The detection noise of 16 atoms $(7 \mathrm{~dB}$ below the projection noise level) is dominated by the photoelectron shot noise on the CCD camera and does not limit the experimental results. Figure 4 (a) presents the Ramsey fringes for the classical case, when the hyperfine level $|g\rangle$ is initially empty. The average fraction $\bar{f}$ is shown as a function of the microwave detuning 
(a)

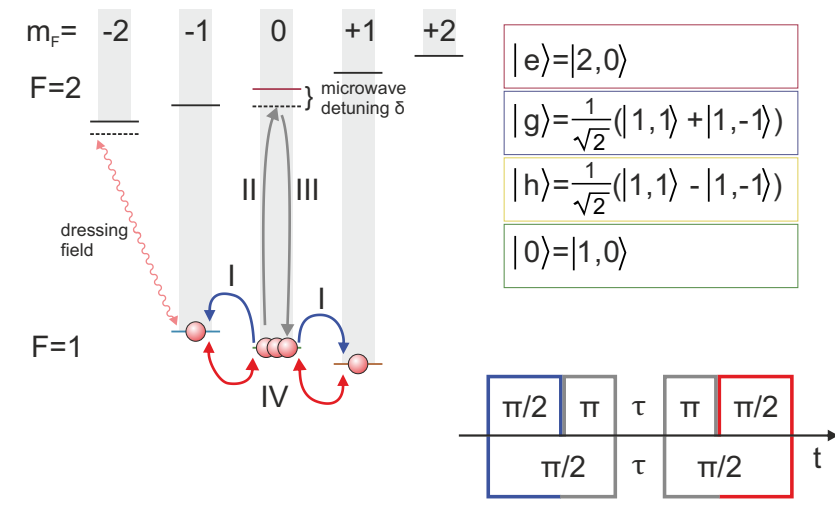

(b)

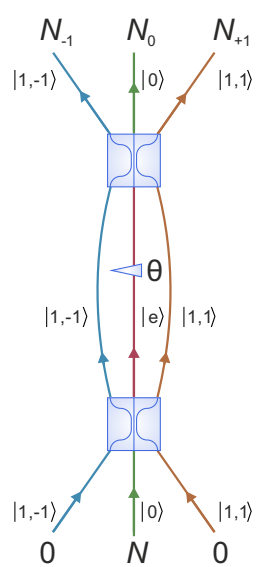

(c)

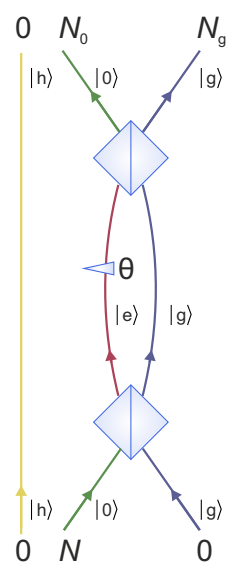

Figure 3. The three-mode interferometer. (a) Hyperfine ground states of ${ }^{87} \mathrm{Rb}$. (I) A Bose-Einstein condensate in the state $|0\rangle=\left|F=1, m_{F}=0\right\rangle$ is coupled to the states $|1, \pm 1\rangle$ by a resonant radio-frequency pulse. The second-order Zeeman shift is compensated by a detuned microwave dressing to the state $|2,-2\rangle$. (II) A microwave pulse with detuning $\delta$ couples the states $|0\rangle=|1,0\rangle$ and $|e\rangle=|2,0\rangle$. The radio-frequency and microwave pulses form an effective $\pi / 2$ pulse between $|1, \pm 1\rangle$ and $|e\rangle$. (III) After a Ramsey evolution time $\tau_{\mathrm{R}}$, a second detuned microwave pulse is applied. (IV) A final radio-frequency pulse coupling $|0\rangle$ and $|1, \pm 1\rangle$ closes the interferometer. (b) The interferometer corresponds to two three-mode beam splitters and a phase shift in between. The total number of atoms $N$ enters the central state $|0\rangle$. A measurement of all three components $N_{-1,0,1}$ after the interferometer allows for an estimation of the phase shift $\theta$. (c) The interferometer can be simplified by introducing the symmetric state $|g\rangle$ and the antisymmetric state $|h\rangle$. The three-mode beam splitters only couple to the symmetric state, thus yielding an effective two-mode interferometer with an unchanged antisymmetric state.

$\delta$ for two Ramsey times $\tau_{\mathrm{R}}$ and is well reproduced by a single-atom model. ${ }^{30}$ The slightly reduced contrast for larger $\tau_{\mathrm{R}}$ stems mainly from the influence of a small radio-frequency detuning as well as magnetic field noise. In addition, the model accounts for the fixed pulse length $\tau_{\mathrm{mw}}$ for all detunings, leading to a small amount of atoms remaining in the $F=2$ manifold $\left(<2.5 \%\right.$ for $\tau_{\mathrm{R}}=250 \mu \mathrm{s}$ and $<8 \%$ for $\tau_{\mathrm{R}}=1000 \mu$ s for up to $2 \mathrm{kHz}$ detuning). In the limit of weak magnetic field gradients, these atoms experience the same spatial separation as the atoms in $F=1$ and thus cannot be discriminated by our absorption imaging. In the following, the pulse length is adjusted such that no atoms remain in the $F=2$ manifold. For small $\tau_{\mathrm{R}}$ the data are in good agreement with the noiseless prediction $\bar{f}=\sin ^{2} \pi \delta \tau$.

The clock performance is evaluated for a vanishing time $\tau_{\mathrm{R}}=0$ between the two detuned microwave pulses (II and III) to minimize technical noise. For a microwave pulse length of $2 \tau_{\mathrm{mw}}=90.4 \mu \mathrm{s}$ and a detuning of $\delta=-5.5 \mathrm{kHz}$ we reach the mid-fringe position $\theta=2 \pi \tau_{\mathrm{mw}} \delta=\pi / 2$, where the slope $\partial \bar{f} / \partial \theta$ has its maximum value $1 / 2$. Here, $\tau_{\mathrm{mw}}$ is chosen such that all atoms return to the state $|0\rangle$. On mid-fringe position, the full interferometer sequence can be described as a single, symmetric beam splitter between $|0\rangle$ and $|g\rangle$. Due to the large number of atoms in the state $|0\rangle$, which act as a local oscillator with a defined phase $\varphi$ in the quantum optics sense, the interferometer sequence presents a standard homodyne measurement of the quadratures in state $|g\rangle$. Therefore, the fluctuations of the interferometer output reflect the quadrature fluctuations: $(\Delta f)^{2}=(\Delta X)^{2} /(2 N) .{ }^{30}$ Here, $X=\frac{1}{\sqrt{2}}\left(e^{-i \varphi} g+e^{i \varphi} g^{\dagger}\right)$ and $P=\frac{1}{i \sqrt{2}}\left(e^{-i \varphi} g-e^{i \varphi} g^{\dagger}\right)$ are quadrature operators of the symmetric state $|g\rangle$, defined in terms of the creation and annihilation operators $g^{\dagger}$ and $g$, respectively, and $\varphi$ is the local oscillator phase. With an initially empty state $|g\rangle$, the quadrature fluctuation is $(\Delta X)^{2}=\frac{1}{2}$. This limits the ideal phase estimation uncertainty to $(\Delta \theta)^{2}=(\Delta f)^{2} /(\partial \bar{f} / \partial \theta)^{2}=1 / N$. In our experiments, we record a value of $(\Delta f)^{2}=1.48 / N$, which is $1.69 \mathrm{~dB}$ above the vacuum limit due to technical noise mainly caused by magnetic field fluctuations. ${ }^{30}$

The sensitivity of our interferometer can surpass the SQL when quadrature fluctuations are squeezed below the vacuum limit, $(\Delta X)^{2}<1 / 2$. We create a squeezed vacuum state by initiating spin dynamics in the BoseEinstein condensate prior to the interferometer sequence. Spin dynamics can be precisely controlled by switching the microwave dressing of the state $|-1\rangle$ to the resonance condition. ${ }^{7}, 31$ In direct analogy to optical parametric 
down conversion, spin dynamics creates pairs of atoms according to the two-mode squeezing Hamiltonian $H=$ $\hbar \Omega\left(a_{+1}^{\dagger} a_{-1}^{\dagger}+a_{+1} a_{-1}\right),{ }^{5,30,32}$ where $a_{ \pm 1}^{\dagger}$ and $a_{ \pm 1}$ are the creation and annihilation operators for atoms in $| \pm 1\rangle$, and $\Omega=2 \pi \times 3.9 \mathrm{~s}^{-1}$ is the spin dynamics rate. Using the operators $g=\left(a_{+1}+a_{-1}\right) / \sqrt{2}$ and $h=\left(a_{+1}-a_{-1}\right) / \sqrt{2}$, this Hamiltonian simplifies to $H=H_{g}-H_{h}$, with $H_{g}=\frac{\hbar \Omega}{2}\left(g^{\dagger} g^{\dagger}+g g\right)$ and the analogous definition for $H_{h}$. Spin dynamics $e^{-i H t / \hbar}=e^{-i r\left(g^{\dagger} g^{\dagger}+g g\right) / 2} \otimes e^{i r\left(h^{\dagger} h^{\dagger}+h h\right) / 2}$, equivalent to a two-axis counter-twisting dynamics ${ }^{33,34}$ $\left(\mathrm{see}^{30}\right)$, can thus be written in terms of the product of usual single-mode quadrature-squeezing operators, ${ }^{35}$ where $r=\Omega t$. We notice that $H_{g}$ and $H_{h}$ rewrite as two-axis counter-twisting Hamiltonians ${ }^{33,34}$ for opportune spin operators, see. ${ }^{30}$ We apply spin dynamics for $32 \mathrm{~ms}$, which creates a mean number $\sinh ^{2} r=0.75$ of atoms in each of the two states. This number is extremely small compared to the total of $N \approx 10^{4}$ atoms, such that the influence of the antisymmetric state to the interferometer signal is negligible and we can approximate $f=\frac{N_{+1}+N_{-1}}{N}=\frac{N_{g}+N_{h}}{N} \approx \frac{N_{g}}{N}$. Even though the symmetric state $|g\rangle$ is only weakly populated, it has a strong influence on the interferometric sensitivity. The squeezing allows for reduced quadrature fluctuations of $(\Delta X)^{2}=\frac{1}{2} e^{-2 r}<\frac{1}{2}$ for $r>0$ at an optimal local oscillator phase $\varphi=\frac{\pi}{4}$. Experimentally, the phase $\varphi$ is adjusted by applying a controlled energy shift with the microwave dressing field for a variable duration $t$ prior to the interferometer sequence.

(a)

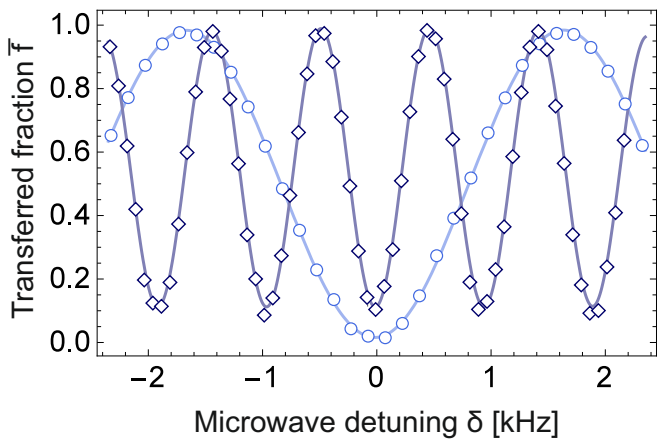

(b)

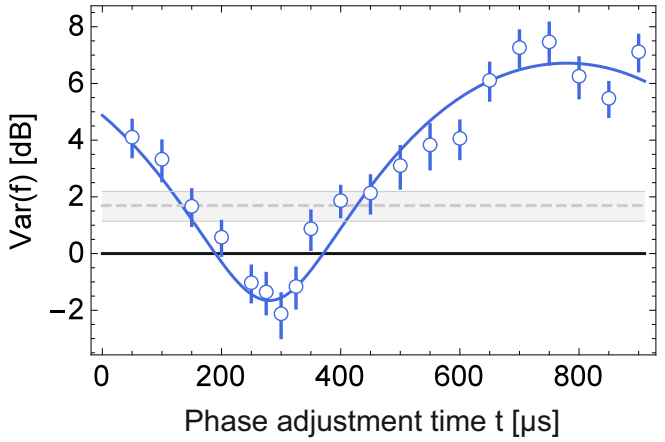

Figure 4. Output of the interferometer. (a) The microwave detuning $\delta$ is varied for different Ramsey times $\tau_{\mathrm{R}}=250 \mu \mathrm{s}$ $(\bigcirc)$ and $\tau_{\mathrm{R}}=1000 \mu \mathrm{s}(\diamond)$. The phase shift, set by the microwave detuning ( $x$-axis) and the evolution time $\tau=\tau_{\mathrm{R}}+\tau_{\mathrm{mw}}$, results in the Ramsey fringes in the transferred fraction. The solid lines $(-,-)$ represent the results of our model. (b) The phase adjustment time $t$ before the interferometer sequence is varied and the corresponding variance of the transferred fraction is recorded with respect to projection noise $(\bigcirc)$. The data is well reproduced by a sinusoidal fit $(-)$ and reaches clearly below projection noise (-). Result of the classical interferometer $\left({ }^{-*}\right)$. The error bars and shaded areas represent the statistical uncertainty of 100 repetitions.

Figure 4 (b) shows the variance of the population imbalance $(\Delta f)^{2}$ as a function of the adjusted phase relation. At an optimum value of $t=300 \mu \mathrm{s}$, a minimal variance of $-2.12_{-.83}^{+.70} \mathrm{~dB}$ below projection noise is reached. The variances are obtained from a total number of 100 repetitions of the experiment per phase adjustment time $t$. All error bars indicate one standard deviation of the statistical uncertainty and are obtained by an unbiased estimation (see Supplements of Ref. ${ }^{7}$ ). Figure 5 (a) shows the fraction $\bar{f}$ as a function of the detuning in the vicinity of the mid-fringe position, as obtained from 390 experimental realizations for each microwave detuning $\delta$. The slope is proportional to the contrast of the interferometer and depends on the coherence properties 


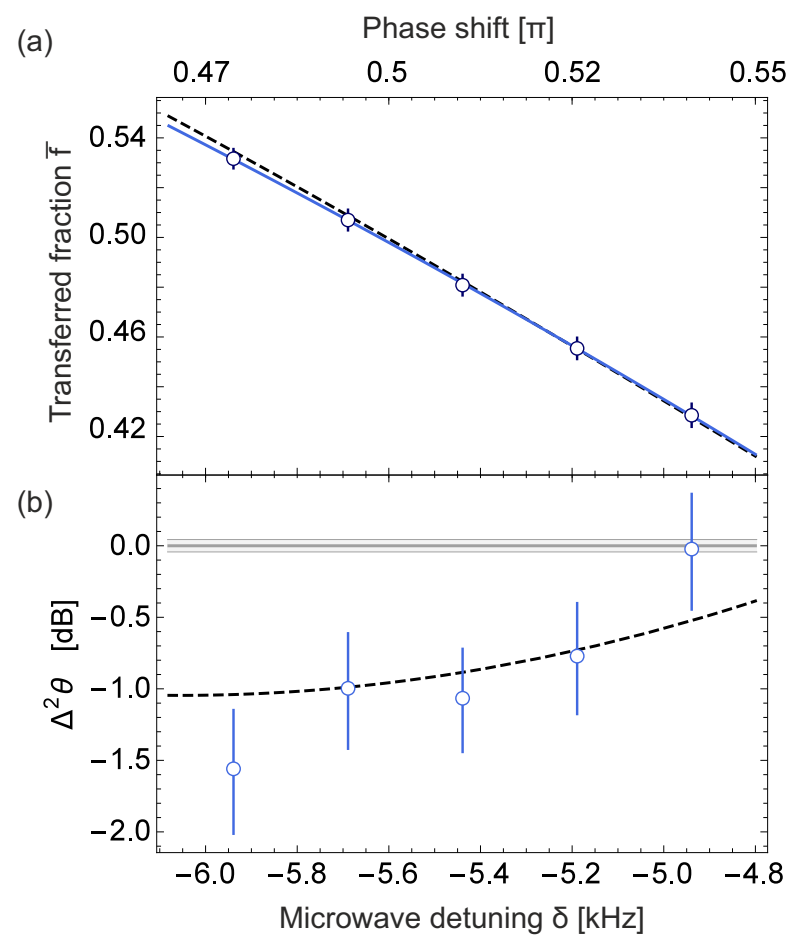

Figure 5. Phase estimation uncertainty. (a) Mean values of the transferred fraction $\bar{f}(\mathrm{O})$ for five different microwave detunings (bottom $x$-axis) and corresponding phase shifts (top $x$-axis) close to the mid-fringe position. The slope of the linear fit $(-)$ is close to the optimum, as represented by our model $(\cdots)$. The error bars represent the (sub- projection noise) statistical uncertainty of 390 repetitions. (b) The phase estimation uncertainties $\Delta^{2} \theta$ normalized to the SQL $(O)$ reach well below the SQL $(-)$. The shaded area represents an upper bound of the uncertainty of the atom number calibration of $1 \%{ }^{36}$ The small detuning dependency of the recorded sensitivity is reproduced by our single-atom model including magnetic field noise $(\cdots)$. The best phase estimation uncertainty of $-1.56_{-.45}^{+.41} \mathrm{~dB}$ below the SQL is reached at a detuning of $-5.9 \mathrm{kHz}$. Error bars are the statistical uncertainty (1 s.d.) of 390 repetitions.

of the input state. A fit (blue solid line) yields a value of $0.48 \mathrm{rad}^{-1}$, which is close to the optimal value of $0.5 \mathrm{rad}^{-1}$. The variances of the population imbalance and the fitted slope yield the phase estimation uncertainty $(\Delta \theta)^{2}=(\Delta f)^{2} /(\partial \bar{f} / \partial \theta)^{2}$ displayed in Fig. 5 (b). At a detuning of $-5.9 \mathrm{kHz},(\Delta \theta)^{2}$ reaches a minimum value $-1.56_{-.45}^{+.41} \mathrm{~dB}$ below the SQL. The two-sample variance, which rejects long term technical drifts and is therefore better suited to estimate the fundamental noise, reaches $-2.05_{-.37}^{+.34} \mathrm{~dB}$ below the SQL.

The interferometric measurements also allow for a reconstruction of the squeezed vacuum state in mode $|g\rangle$. The Wigner function in $X$-P-space after the optimal phase adjustment time of $300 \mu$ s is obtained from an inverse Radon transformation (see Fig. 6 (a) and ${ }^{30}$ ). Its profile is very close to the expected Gaussian distribution, and is characterized by the squeezed and anti-squeezed widths along the $X$ and $P$ directions, respectively. While this single-mode picture successfully describes the physics of our experiments, it can equivalently be described by spin squeezing of the usual two-mode pseudo-spin operators, as visualized on the multi-particle Bloch sphere in Fig. 6 (b). It is worth noting, that these collective pseudo-spin operators are identical to the SU(2) subspaces exhibiting spin-nematic squeezing reported in Ref. ${ }^{37}$ Furthermore, the created squeezed vacuum state can also be employed for phase sensing in a nonlinear interferometer scheme of the $\mathrm{SU}(1,1)$ type. ${ }^{38,39}$

In summary, our experiments present the first proof-of-principle implementation of squeezed vacuum in an atomic microwave clock. Microwave fountain clocks, providing the realization of the SI second, are currently limited by the SQL. ${ }^{22,40-42}$ In combination with the recently developed sources of Bose-Einstein condensed atoms with small densities ${ }^{43,44}$ and high repetition rates ${ }^{45}$ our results pave the way for the development of a new generation of atomic microwave clocks operating beyond the SQL. ${ }^{30}$ Our method is particularly robust during state preparation. In contrast to existing proposals, it avoids the generation of entangled states with a 
(a)

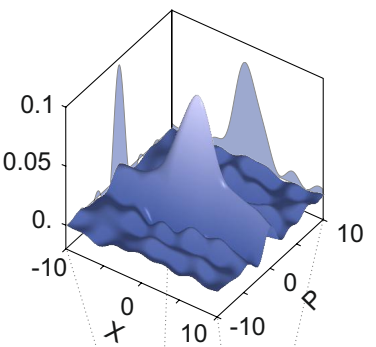

(b)

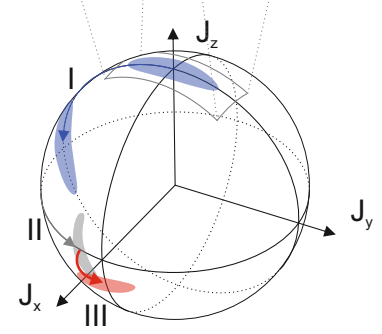

Figure 6. Reconstructed Wigner function and Bloch sphere representation. (a) The data presented in Fig. 4 (b) is used to reconstruct the two-dimensional Wigner function in the $X$ - $P$-space. Here, the Wigner function of the state after the optimal phase adjustment time of $t=300 \mu \mathrm{s}$ is shown. (b) The interferometer is illustrated on the multi-particle Bloch sphere for the states $|g\rangle$ and $|e\rangle$, in terms of the pseudo-spin operators $J_{x}=\frac{1}{2}\left(e^{\dagger} g+g^{\dagger} e\right), J_{y}=\frac{1}{2 i}\left(e^{\dagger} g-g^{\dagger} e\right)$, $J_{z}=\frac{1}{2}\left(e^{\dagger} e-g^{\dagger} g\right)$. The employed squeezed vacuum corresponds to an elliptical uncertainty disk with variable orientation angle depending on the phase adjustment time $t$. An optimal orientation angle, as shown, allows for a measurement of the transferred fraction with a sub-projection-noise uncertainty. The two radio-frequency pulses generate rotations around the $J_{x}$-axis (I/III). The phase shift corresponds to a rotation around the $J_{z}$-axis (II).

symmetric population of the two hyperfine levels, which is plagued by two-body losses in the excited hyperfine state. The limitations of our method for sub-SQL interferometry have not been reached yet: besides overcoming technical restrictions, it has been shown ${ }^{46}$ that an optimized version of the present interferometric scheme can reach the ultimate Heisenberg limit of phase sensitivity $\Delta \theta=1 / N$.

\section{ACKNOWLEDGMENTS}

We acknowledge support from the Centre for Quantum Engineering and Space-Time Research (QUEST) and from the Deutsche Forschungsgemeinschaft through CRC 1227 (DQ-mat), project A02, Research Training Group 1729 , and the EMRP project QESOCAS. The EMRP is jointly funded by the EMRP participating countries within EURAMET and the European Union. J. A. acknowledges support by the Lundbeck Foundation

\section{REFERENCES}

[1] LIGO Scientific Collaboration and Virgo Collaboration, "Observation of gravitational waves from a binary black hole merger," Phys. Rev. Lett. 116, 061102 (Feb 2016).

[2] Huntemann, N., Lipphardt, B., Tamm, C., Gerginov, V., Weyers, S., and Peik, E., "Improved limit on a temporal variation of $m_{p} / m_{e}$ from comparisons of $\mathrm{Yb}^{+}$and Cs atomic clocks," Phys. Rev. Lett. 113, 210802 (Nov 2014).

[3] Godun, R. M., Nisbet-Jones, P. B. R., Jones, J. M., King, S. A., Johnson, L. A. M., Margolis, H. S., Szymaniec, K., Lea, S. N., Bongs, K., and Gill, P., "Frequency ratio of two optical clock transitions in ${ }^{171} \mathrm{yb}^{+}$and constraints on the time variation of fundamental constants," Phys. Rev. Lett. 113, 210801 (Nov 2014).

[4] Caves, C. M., "Quantum-mechanical noise in an interferometer," Phys. Rev. D 23, 1693-1708 (Apr 1981).

[5] Klempt, C., Topic, O., Gebreyesus, G., Scherer, M., Henninger, T., Hyllus, P., Ertmer, W., Santos, L., and Arlt, J. J., "Parametric amplification of vacuum fluctuations in a spinor condensate," Phys. Rev. Lett. 104, 195303 (May 2010). 
[6] Scherer, M., Lücke, B., Gebreyesus, G., Topic, O., Deuretzbacher, F., Ertmer, W., Santos, L., Arlt, J. J., and Klempt, C., "Spontaneous breaking of spatial and spin symmetry in spinor condensates," Phys. Rev. Lett. 105, 135302 (Sep 2010).

[7] Lücke, B., Peise, J., Vitagliano, G., Arlt, J., Santos, L., Tóth, G., and Klempt, C., "Detecting multiparticle entanglement of Dicke states," Phys. Rev. Lett. 112, 155304 (Apr 2014).

[8] Appel, J., Windpassinger, P. J., Oblak, D., Hoff, U. B., Kærgaard, N., and Polzik, E. S., "Mesoscopic atomic entanglement for precision measurements beyond the standard quantum limit," Proc. Natl. Acad. Sci. U. S. A. 106(27), 10960 (2009).

[9] Wasilewski, W., Jensen, K., Krauter, H., Renema, J. J., Balabas, M. V., and Polzik, E. S., "Quantum noise limited and entanglement-assisted magnetometry," Phys. Rev. Lett. 104, 133601 (Mar 2010).

[10] Schleier-Smith, M. H., Leroux, I. D., and Vuletić, V., "States of an ensemble of two-level atoms with reduced quantum uncertainty," Phys. Rev. Lett. 104, 073604 (Feb 2010).

[11] Bohnet, J. G., Cox, K. C., Norcia, M. A., Weiner, J. M., Chen, Z., and Thompson, J. K., "Reduced spin measurement back-action for a phase sensitivity ten times beyond the standard quantum limit," Nat. Photon. 8, 731-736 (July 2014).

[12] Behbood, N., Ciurana, F. M., Colangelo, G., Napolitano, M., Tóth, G., Sewell, R. J., and Mitchell, M. W., "Generation of macroscopic singlet states in a cold atomic ensemble," Phys. Rev. Lett. 113, 093601 (Aug 2014).

[13] Hosten, O., Engelsen, N. J., Krishnakumar, R., and Kasevich, M. A., "Measurement noise 100 times lower than the quantum-projection limit using entangled atoms," Nature 529, 505 (2016).

[14] Gross, C., Zibold, T., Nicklas, E., Estève, J., and Oberthaler, M. K., "Nonlinear atom interferometer surpasses classical precision limit," Nature 464, 1165-1169 (Apr. 2010).

[15] Riedel, M., Böhi, P., Li, Y., Hänsch, T., Sinatra, A., and Treutlein, P., "Atom-chip-based generation of entanglement for quantum metrology," Nature 464, 1170 (Apr. 2010).

[16] Strobel, H., Muessel, W., Linnemann, D., Zibold, T., Hume, D. B., Pezzè, L., Smerzi, A., and Oberthaler, M. K., "Fisher information and entanglement of non-Gaussian spin states," Science 345(6195), 424-427 (2014).

[17] Lücke, B., Scherer, M., Kruse, J., Pezzé, L., Deuretzbacher, F., Hyllus, P., Topic, O., Peise, J., Ertmer, W., Arlt, J., Santos, L., Smerzi, A., and Klempt, C., "Twin matter waves for interferometry beyond the classical limit," Science 334, 773 (Nov. 2011).

[18] The LIGO Scientific Collaboration, "A gravitational wave observatory operating beyond the quantum shotnoise limit," Nature Phys. 7, 962-965 (2011).

[19] Aasi, J., Abadie, J., Abbott, B. P., Abbott, R., Abbott, T. D., Abernathy, M. R., Adams, C., Adams, T., Addesso, P., Adhikari, R. X., and et al., "Enhanced sensitivity of the LIGO gravitational wave detector by using squeezed states of light," Nature Photon. 7, 613-619 (Aug. 2013).

[20] Weyers, S., Gerginov, V., Nemitz, N., Li, R., and Gibble, K., "Distributed cavity phase frequency shifts of the caesium fountain PTB-CSF2," Metrologia 49(1), 82 (2012).

[21] Bloom, B., Nicholson, T., Williams, J., Campbell, S., Bishof, M., Zhang, X., Zhang, W., Bromley, S., and Ye, J., "An optical lattice clock with accuracy and stability at the $10^{-18}$ level," Nature 506(7486), 71-75 (2014).

[22] Santarelli, G., Laurent, P., Lemonde, P., Clairon, A., Mann, A. G., Chang, S., Luiten, A. N., and Salomon, C., "Quantum projection noise in an atomic fountain: A high stability cesium frequency standard," Phys. Rev. Lett. 82, 4619-4622 (Jun 1999).

[23] Ramsey, N. F., "A new molecular beam resonance method," Phys. Rev. 76, 996 (Oct 1949).

[24] Sewell, R. J., Koschorreck, M., Napolitano, M., Dubost, B., Behbood, N., and Mitchell, M. W., "Magnetic sensitivity beyond the projection noise limit by spin squeezing," Phys. Rev. Lett. 109, 253605 (Dec 2012).

[25] Muessel, W., Strobel, H., Linnemann, D., Hume, D. B., and Oberthaler, M. K., "Scalable spin squeezing for quantum-enhanced magnetometry with bose-einstein condensates," Phys. Rev. Lett. 113, 103004 (Sep 2014).

[26] Ockeloen, C. F., Schmied, R., Riedel, M. F., and Treutlein, P., "Quantum metrology with a scanning probe atom interferometer," Phys. Rev. Lett. 111, 143001 (Oct 2013). 
[27] Louchet-Chauvet, A., Appel, J., Renema, J. J., Oblak, D., Kjaergaard, N., and Polzik, E. S., "Entanglementassisted atomic clock beyond the projection noise limit," New Journal of Physics 12(6), 065032 (2010).

[28] Meyer, V., Rowe, M. A., Kielpinski, D., Sackett, C. A., Itano, W. M., Monroe, C., and Wineland, D. J., "Experimental demonstration of entanglement-enhanced rotation angle estimation using trapped ions," Phys. Rev. Lett. 86, 5870-5873 (Jun 2001).

[29] LIGO Scientific Collaboration, "A gravitational wave observatory operating beyond the quantum shot-noise limit," Nature Physics 7(12), 962-965 (2011).

[30] See Supplemental Material [url], which includes Refs. [38-45].

[31] Klempt, C., Topic, O., Gebreyesus, G., Scherer, M., Henninger, T., Hyllus, P., Ertmer, W., Santos, L., and Arlt, J. J., "Multiresonant spinor dynamics in a Bose-Einstein condensate," Phys. Rev. Lett. 103, 195302 (Nov 2009).

[32] Duan, L.-M., Cirac, J. I., and Zoller, P., "Quantum entanglement in spinor Bose-Einstein condensates," Phys. Rev. A 65, 033619 (Feb 2002).

[33] Kitagawa, M. and Ueda, M., "Squeezed spin states," Phys. Rev. A 47, 5138-5143 (Jun 1993).

[34] Opatrný, T., "Twisting tensor and spin squeezing," Phys. Rev. A 91, 053826 (May 2015).

[35] Scully, M. and Zubairy, M., [Quantum optics], Cambridge Univ Pr (1997).

[36] Peise, J., Kruse, I., Lange, K., Lücke, B., Pezzè, L., Arlt, J., Ertmer, W., Hammerer, K., Santos, L., Smerzi, A., and Klempt, C., "Satisfying the Einstein-Podolsky-Rosen criterion with massive particles," Nat. Commun. 6(8984) (2015).

[37] Hamley, C. D., Gerving, C. S., Hoang, T. M., Bookjans, E. M., and Chapman, M. S., "Spin-nematic squeezed vacuum in a quantum gas," Nature Phys. 8, 305 (Feb. 2012).

[38] Linnemann, D., Strobel, H., Muessel, W., Schulz, J., Lewis-Swan, R. J., Kheruntsyan, K. V., and Oberthaler, M. K., "Quantum-enhanced sensing based on time reversal of nonlinear dynamics," Phys. Rev. Lett. 117, 013001 (Jun 2016).

[39] Gabbrielli, M., Pezzè, L., and Smerzi, A., "Spin-mixing interferometry with bose-einstein condensates," Phys. Rev. Lett. 115, 163002 (Oct 2015).

[40] Millo, J., Abgrall, M., Lours, M., English, E. M. L., Jiang, H., Guéna, J., Clairon, A., Tobar, M. E., Bize, S., Le Coq, Y., and Santarelli, G., "Ultralow noise microwave generation with fiber-based optical frequency comb and application to atomic fountain clock," Appl. Phys. Lett. 94(14) (2009).

[41] Weyers, S., Lipphardt, B., and Schnatz, H., "Reaching the quantum limit in a fountain clock using a microwave oscillator phase locked to an ultrastable laser," Phys. Rev. A 79, 031803 (Mar 2009).

[42] Dobrev, G., Gerginov, V., and Weyers, S., "Loading a fountain clock with an enhanced low-velocity intense source of atoms," Phys. Rev. A 93, 043423 (Apr 2016).

[43] Müntinga, H., Ahlers, H., Krutzik, M., Wenzlawski, A., Arnold, S., Becker, D., Bongs, K., Dittus, H., Duncker, H., Gaaloul, N., Gherasim, C., Giese, E., Grzeschik, C., Hänsch, T. W., Hellmig, O., Herr, W., Herrmann, S., Kajari, E., Kleinert, S., Lämmerzahl, C., Lewoczko-Adamczyk, W., Malcolm, J., Meyer, N., Nolte, R., Peters, A., Popp, M., Reichel, J., Roura, A., Rudolph, J., Schiemangk, M., Schneider, M., Seidel, S. T., Sengstock, K., Tamma, V., Valenzuela, T., Vogel, A., Walser, R., Wendrich, T., Windpassinger, P., Zeller, W., van Zoest, T., Ertmer, W., Schleich, W. P., and Rasel, E. M., "Interferometry with Bose-Einstein condensates in microgravity," Phys. Rev. Lett. 110, 093602 (Feb 2013).

[44] Dickerson, S. M., Hogan, J. M., Sugarbaker, A., Johnson, D. M. S., and Kasevich, M. A., "Multiaxis inertial sensing with long-time point source atom interferometry," Phys. Rev. Lett. 111, 083001 (Aug 2013).

[45] Rudolph, J., Herr, W., Grzeschik, C., Sternke, T., Grote, A., Popp, M., Becker, D., Müntinga, H., Ahlers, H., Peters, A., Lämmerzahl, C., Sengstock, K., Gaaloul, N., Ertmer, W., and Rasel, E. M., "A high-flux BEC source for mobile atom interferometers," New J. Phys. 17(6), 065001 (2015).

[46] Pezzé, L. and Smerzi, A., "Mach-Zehnder interferometry at the Heisenberg limit with coherent and squeezedvacuum light," Phys. Rev. Lett. 100, 073601 (Feb 2008). 